\title{
Positive association between preoperative lymphocyte-to- monocyte ratio and risk of the status of positive surgical margins by prostate cancer: results in 497 consecutive patients treated only by radical prostatectomy
}

\author{
Jiatong Zhou, Ranlu Liu \\ Department of Urology, The Second Hospital of Tianjin Medical University, Tianjin, China \\ Contributions: (I) Conception and design: All authors; (II) Administrative support: All authors; (III) Provision of study materials or patients: All \\ authors; (IV) Collection and assembly of data: All authors; (V) Data analysis and interpretation: J Zhou; (VI) Manuscript writing: All authors; \\ (VII) Final approval of manuscript: All authors. \\ Correspondence to: Ranlu Liu. Department of Urology, The Second Hospital of Tianjin Medical University, No. 23, Pingjiang Road, Hexi District, \\ Tianjin 300211, China. Email: 16622080858@163.com.
}

Background: Positive surgical margins (PSM) is one of the most important factors affecting the prognosis of prostate cancer ( $\mathrm{PCa}$ ) patients after radical prostatectomy (RP). Although some studies have found the preoperative systematic inflammation-based scores the neutrophil-to-lymphocyte ratio (NLR), the plateletto-lymphocyte ratio (PLR), lymphocyte-to-monocyte ratio (LMR) can predict the incidence and prognosis of $\mathrm{PCa}$, few studies have explored the predictive value of preoperative systematic inflammation-based scores on the PSMs for PCa patients after RP.

Methods: From June 2014 to September 2020 a total of 497 patients underwent RP at our institution. Blood samples from all patients were collected within one week before surgery. Preoperative clinical characteristics including age, body mass index (BMI), prostate-specific antigen (PSA), and biopsy Gleason sum (BGS) were assessed. Postoperatively pathological specimens were assessed for pathological Gleason sum (PGS), pathological stage, and margin status.

Results: In the multivariable analysis including preoperative variables, PSA and LMR were the independent predictive factors for PSM (OR: 2.817; 95\% CI, 1.836-4.320, P<0.001; OR: 1.124; 95\% CI, 1.018-1.240, $\mathrm{P}=0.021$. Considering pre-, intra-, and postoperative variables, BGS, perineural invasion, seminal vesicle invasion (SVI), pathologic Gleason sum (PGS) combined, were associated with increased risk of PSM in the univariable analysis $(\mathrm{P}<0.001$ for all variables). However, in the multivariable analysis, perineural invasion (OR: 2.672; 95\% CI, 1.649-4.330; $\mathrm{P}<0.001$ ), PGS (OR: 2.52; 95\% CI, 1.556-4.082; $\mathrm{P}<0.001)$ were independent predictive factors for the incidence of PSM. Finally, LMR was shown to be an independent predictive factor (OR: 0.881; 95\% CI, 0.779-0.996; $\mathrm{P}=0.043$ ) for apical PSMs, with increasing LMR predicting the lower incidence of apex location. And we also found that LMR was an independent factor that predicts multifocal positive margins (OR: 1.179; 95\% CI, 1.023-1.358; P=0.023).

Conclusions: Preoperative LMR could be used as an independent predictor to predict the incidence of PSMs after RP. And Considering pre-, intra-, and postoperative variables, we also found that preoperative LMR could predict the occurrence of apical and multifocal PSMs.

Keywords: Prostate cancer (PCa); positive surgical margins (PSM); lymphocyte-to-monocyte ratio (LMR)

Submitted Nov 20, 2020. Accepted for publication Jan 15, 2021.

doi: $10.21037 /$ tau-20-1447

View this article at: http://dx.doi.org/10.21037/tau-20-1447 


\section{Introduction}

Prostate cancer $(\mathrm{PCa})$ is the most common male malignant tumor and the second leading cause of male cancer-related mortality (1). At present, radical prostatectomy (RP) is the gold standard treatment for localized PCa. With the development of surgical techniques and technology, it has gradually shifted from open radical prostatectomy (ORP) to laparoscopic radical prostatectomy (LRP). The development of technology has made the surgical treatment of localized PCa safer and more minimally invasive, and greatly improved the quality of life and prognosis of $\mathrm{PCa}$ patients (2).

Although patients with PCa can obtain a clinical cure or a good prognosis through RP, there are still some important factors that can lead to tumor recurrence and progression.

According to previous literature reports, the incidence rate of positive surgical margins (PSM) after RP is approximately $10-35 \%$, and PSMs are considered to be one of the most significant indicators for cancer recurrence and poor prognosis in PCa patients (3-5). Compared with patients with negative surgical margins, PSMs may not only cause tumor recurrence after surgery but also cause mental stress to PCa patients (6). Recent studies have confirmed that tumor-related inflammation affects the malignancy of tumors, including tumor proliferation and survival, angiogenesis, metastasis, and treatment response (7). In some studies, some cancer-related inflammatory indexes including neutrophil-to-lymphocyte ratio (NLR), the platelet-to-lymphocyte ratio (PLR), lymphocyte-tomonocyte ratio (LMR), and prognostic nutritional index play an important role in predicting the prognosis of different tumors, these are serum-based and not pathology review based (8-12). However, few articles reported the association between tumor-related inflammation scores and PSMs status after RP. Therefore, we performed this study to explore whether these inflammation scores can be used as important predictors to predict PSMs status. We present the following article in accordance with the STROBE reporting checklist (available at http://dx.doi.org/10.21037/ tau-20-1447).

\section{Methods}

\section{Patients}

This study reviewed all patients from June 2014 to September 2020, 497 patients of PCa who underwent RP in the second hospital of Tianjin Medical University were included. The retrospective data were collected following criteria: no neoadjuvant or adjuvant treatment, distant metastasis confirmed by surgery or imaging. Excluded criteria: Patients with genetic immunodeficiency, acquired immunodeficiency syndrome, immunosuppressive drugs, and other immune deficiency diseases; patients with hematological diseases; long-term immunotherapy; patients with other malignant tumors; incomplete record. The surgical techniques for RP with or without lymph node dissection differed among patients: open RP or laparoscopic RP. Both interventions were conducted by two experienced surgical teams. The protocol for processing RP specimens was similar across sites, the remaining specimen was sectioned transversely at intervals of $3-5 \mathrm{~mm}$ formalin-fixed and embedding all sections for analysis. PSMs was defined as the appearance of tumor cells on the surface of the surgical specimen and were categorized into five groups based on their locations: apex, proximal (bladder neck), peripheral, focal, and multifocal. Determine the occurrence and location of positive margins based on previous studies (13). A pathologist performed all histopathological diagnoses and prepared prostate specimens using the same method during the research. Focal positive margins are considered to be only a single positive location in the surgical specimen, and multifocal positive margins are defined as more than 1 positive location in the surgical specimen. The study was conducted in accordance with the Declaration of Helsinki (as revised in 2013). The study was approved by the Regional Ethical Review Board in Tianjin medical university second hospital and individual consent for this retrospective analysis was waived.

\section{Definitions and of LMR, NLR, PLR, prognostic nutritional index}

The definitions of prognostic nutritional index, NLR, and PLR were shown as follows: prognostic nutritional index $=$ albumin $(\mathrm{g} / \mathrm{L})+5 \times$ total lymphocyte counts $\left(10^{9} / \mathrm{L}\right)$; $\mathrm{SII}=$ platelet $\times$ neutrophil/lymphocyte counts; NLR = neutrophil/lymphocyte counts; and PLR = platelet $/$ lymphocyte counts. Blood samples of all patients were collected within one weeks before surgery.

\section{Statistical analysis}

The entire statistical process was performed with SPSS 22.0 software. Measurement values for continuous variables are expressed as the median (range). Qualitative data were 
expressed as a percentage (\%) and analyzed by the $\chi^{2}$ test. The receiver operating characteristic (ROC) curve was plotted by referring to the sensitivity $v$ s. 1-specificity of the LMR level. The areas under the curve (AUCs) and cut-off values were also calculated. Sensitivity, specificity was used to estimate the value of LMR based on the cut-off value. Univariate and multivariate logistic regression analysis was used to screen out the independent risk factors for the incidence, location of PSMs. All analyses are bilateral analysis, $\mathrm{P}<0.05$ has statistical significance.

\section{Results}

Patient Features are shown in Table 1. A total of 497 patients were enrolled in our study, from June 2014 to September 2020. The overall PSMs rate was $53.7 \%$ (267 of 497). The distribution of these PSMs by location was 69.3\% (185 of 267 ) in the apex, $23.3 \%$ (116 of 267) peripheral, $34.5 \%$ (92 of 267) proximal. Focal PSM was 59.2\% (158 of 267) and multifocal PSM was 40.8\% (104 of 267).

\section{Model 1: preoperative and postoperative factors in PSMs}

In preoperative factors, no association was observed in the univariable analysis (Table 2) between PSM and age ( $\mathrm{P}=0.904)$, BMI ( $\mathrm{P}=0.441)$, NLR ( $\mathrm{P}=0.44)$, PLR ( $\mathrm{P}=0.116)$, LMR ( $\mathrm{P}=0.074)$, prognostic nutritional index $(\mathrm{P}=0.46)$. In the multivariable analysis including preoperative variables, LMR was a significant predictive factor, with higher PSM rates (OR: 1.124; $\mathrm{P}<0.021$ ) and also in PSA (OR: 2.817; $\mathrm{P}<0.001$ ) (Table 2). As for postoperative pathological factors, perineural invasion, SVI and pathologic Gleason sum (PGS) were associated with increased risk of PSM in the univariable analysis $(\mathrm{P}<0.001$ for all variables; Table 2$)$. In the multivariable analysis, perineural invasion $(\mathrm{P}<0.001)$ and PGS $(\mathrm{P}<0.001)$ were the only independent predictive factors for PSMs.

\section{Model 2: positive surgical margin location}

Tables 3-5 summarize clinical and pathologic features according to the location of PSMs including apex, peripheral, proximal. LMR, analyzed as a continuous variable, was shown to be an independent predictive factor for apical PSMs, with increasing BMI predicting the lower incidence of apex location (OR: $0.881 ; \mathrm{P}=0.043$ ). ROC curve indicated that a LMR of 7.01 had maximum Youden index value (Figure 1) (sensitivity $=89.7 \%$, Specificity
$=22 \%$ ). The higher the preoperative LMR value, the lower the risk of the positive apex. Regarding peripheral PSM location, PSA (OR: 2.209; $\mathrm{P}=0.006$ ) and perineural invasion (OR: 1.934; $\mathrm{P}=0.014$ ) were determined to be significantly predictive factors, which predicts the incidence risk of peripheral location.

\section{Model 3: focal and multifocal positive margins}

In univariate analysis (Table 6), PSA (OR 2.721; $\mathrm{P}<0.001)$, perineural invasion (OR 2.452; $\mathrm{P}<0.001$ ), SVI (OR 2.714; $\mathrm{P}=0.001)$, $\mathrm{PGS}(\mathrm{OR} 2.4 ; \mathrm{P}<0.001)$ associated with the risk of focal surgical margins. In multivariate analysis (Table 6 ), PSA (OR 2.374; $\mathrm{P}<0.001$ ), perineural invasion (OR 2.349; $\mathrm{P}=0.002$ ), PGS (OR 1.78; $\mathrm{P}=0.039$ ) were independent factors that associated with the risk of focal PSMs. And in the multivariate analysis presented in Table 7, LMR (OR 1.179; $\mathrm{P}=0.023$ ), PSA (OR 3.5; $\mathrm{P}<0.001$ ), perineural invasion (OR 3.446; $\mathrm{P}<0.001$ ), $\mathrm{PGS}$ (OR 3.931; $\mathrm{P}<0.001$ ) were significant with the risk of multifocal PSMs.

\section{Discussion}

PSMs after RP is considered to be one of the important factors leading to postoperative tumor recurrence and local progression, and it is also a key factor in determining whether PCa patients should proceed with the second treatment (14). With the development of surgical techniques and instruments, the probability of PSMs after $\mathrm{RP}$ is decreasing. However, in high-risk PCa patients, the incidence rate of PSMs after RP remains relatively stable (15). There are two main causes for the incidence risk of PSMs, including tumor progression and the experience of the surgeon. With the development of the current surgical technique, the surgical technique of the surgeon is constantly improving. In addition to the surgical experience, the more important reason is the biological behavior of the tumor cell, which is an independent risk factor that affects the PSMs $(14,16)$. Patel et al. performed a muti-institutional study and demonstrated that the apex of the prostate is the most common site for PSMs after robotic-assisted RP, and pathological staging and preoperative PSA are the most important independent risk factors for predicting positive margins (17). Yossepowitch et al. reported that the incidence of PSMs is strongly influenced by the surgeon's experience irrespective of the surgical approach through evidence synthesis and they also thought PSMs are associated with a twofold increased hazard of biochemical relapse, but 
Table 1 Association between individual categorical and continuous variables with positive surgical margins

\begin{tabular}{|c|c|c|c|c|}
\hline Variables & Population $(n=497)$ & \multicolumn{2}{|c|}{ Surgical margin } & $P$ value \\
\hline Age, years, median (IQR) & 68 [63-73] & 68 [63-72] & 67 [63-73] & 0.904 \\
\hline BMI, kg/m², median (IQR) & $25.14(23.5-27)$ & $24.97(23.53-26.83)$ & $25.2(23.38-27.16)$ & 0.442 \\
\hline Diabetes, (\%) & & & & 0.238 \\
\hline Present & $77(15.5)$ & $39(17.0)$ & $38(14.2)$ & \\
\hline Hypertension, (\%) & & & & 0.503 \\
\hline Absent & $254(51.1)$ & $118(51.3)$ & $136(50.9)$ & \\
\hline Present & $243(48.9)$ & $112(48.7)$ & $131(49.1)$ & \\
\hline PSA, (\%) & & & & $<0.001^{*}$ \\
\hline$<20 \mathrm{ng} / \mathrm{mL}$ & $314(63.2)$ & $179(77.8)$ & $135(50.6)$ & \\
\hline$\geq 20 \mathrm{ng} / \mathrm{mL}$ & $183(36.8)$ & $51(22.2)$ & $132(49.4)$ & \\
\hline Serum albumin, g/L, median (IQR) & 44.7 (41.7-47.2) & $44.56(42-47.05)$ & $44.8(41.4-47.5)$ & 0.795 \\
\hline Serum neutrophil, $10^{\circ} / \mathrm{L}$, median (IQR) & $3.6(2.98-4.42)$ & $3.58(2.98-4.4)$ & $3.6(2.99-4.46)$ & 0.896 \\
\hline Serum lymphocyte, $10^{9} / \mathrm{L}$, median (IQR) & $1.79(1.44-2.27)$ & $1.78(1.37-2.26)$ & $1.79(1.47-2.27)$ & 0.132 \\
\hline Serum monocyte, $10^{\circ} / \mathrm{L}$, median (IQR) & $0.37(0.31-0.45)$ & $0.38(0.3-0.45)$ & $0.37(0.31-0.46)$ & 0.828 \\
\hline Biopsy Gleason sum, n (\%) & & & & $<0.001^{*}$ \\
\hline$<8$ & $292(58.8)$ & $155(67.4)$ & $137(51.3)$ & \\
\hline$\geq 8$ & $205(41.2)$ & $75(32.6)$ & $130(48.7)$ & \\
\hline Perineural invasion, n (\%) & & & & $<0.001^{*}$ \\
\hline Absent & $375(75.5)$ & $197(85.7)$ & $178(66.7)$ & \\
\hline Present & $122(24.5)$ & $33(14.3)$ & 89 (33.3) & \\
\hline SVI, n (\%) & & & & $<0.001^{*}$ \\
\hline Absent & 414 (83.3) & $211(91.7)$ & $203(76.4)$ & \\
\hline \multicolumn{5}{|l|}{ Surgical approach, n (\%) } \\
\hline ORP & $28(5.6)$ & $14(50.0)$ & $14(50.0)$ & 0.415 \\
\hline LRP & $469(94.4)$ & $216(46.1)$ & $253(53.9)$ & \\
\hline Present & $83(16.7)$ & $19(8.3)$ & $64(23.6)$ & \\
\hline
\end{tabular}

Table 1 (continued) 
Table 1 (continued)

\begin{tabular}{|c|c|c|c|c|}
\hline Variables & Population $(n=497)$ & \multicolumn{2}{|c|}{ Surgical margin } & $P$ value \\
\hline \multicolumn{5}{|l|}{ Pathologic stage } \\
\hline Organ confined: pT1/pT2, no. (\%) & $211(42.4)$ & $211(91.7)$ & 0 & \\
\hline Non-organ confined: pT3a, no. (\%) & $200(40.2)$ & 0 & $200(74.9)$ & \\
\hline Non-organ confined: pT4, no. (\%) & $8(1.7)$ & 0 & $8(4)$ & \\
\hline Pathologic Gleason sum, no. (\%) & & & & $<0.001^{*}$ \\
\hline$<8$ & $308(62.0)$ & $176(76.5)$ & $132(49.4)$ & \\
\hline$\geq 8$ & $189(38.0)$ & $54(23.5)$ & $135(50.6)$ & \\
\hline Apical & $185(37.2)$ & & $185(69.3)$ & \\
\hline Peripheral & $116(23.3)$ & & $116(23.3)$ & \\
\hline Proximal & $92(18.5)$ & & $92(34.5)$ & \\
\hline \multicolumn{5}{|l|}{ Number of PSM, no. (\%) } \\
\hline Focal positive (single) & $158(31.8)$ & & $158(59.2)$ & \\
\hline Multifocal positive (more than 1) & $109(21.9)$ & & $109(40.8)$ & \\
\hline
\end{tabular}

*, statistically significant. BMI, body mass index; PSA, prostate specific antigen; NLR, neutrophil to lymphocyte ratio; PLR, platelets to lymphocyte ratio; LMR, lymphocyteto monocyte ratio; SVI, seminal vesicle invasion; ORP, open radical prostatectomy.

Table 2 Univariate and Multivariate analysis for positive surgical margins

\begin{tabular}{|c|c|c|c|c|c|c|}
\hline Variables & \multicolumn{3}{|c|}{ Univariate analysis } & \multicolumn{3}{|c|}{ Multivariate analysis } \\
\hline Age & 0.998 & $0.973-1.025$ & 0.904 & & & \\
\hline BMI & 1.025 & $0.963-1.091$ & 0.441 & & & \\
\hline PSA & 3.432 & $2.317-5.083$ & $<0.001^{*}$ & 2.817 & $1.836-4.32$ & $<0.001^{\star}$ \\
\hline PLR & 0.997 & $0.994-1.001$ & 0.116 & & & \\
\hline LMR & 1.086 & $0.992-1.189$ & 0.074 & 1.124 & $1.018-1.24$ & $0.021^{\star}$ \\
\hline Prognostic nutritional index & 1.013 & $0.979-1.047$ & 0.46 & & & \\
\hline Biopsy Gleason sum ( $\geq 8$ vs. <8) & 1.961 & $1.361-2.826$ & $<0.001^{*}$ & 0.902 & $0.566-1.439$ & 0.665 \\
\hline Pathologic Gleason sum ( $\geq 8$ vs. $<8$ ) & 3.333 & $2.261-4.913$ & $<0.001^{*}$ & 2.52 & $1.556-4.082$ & $<0.001^{\star}$ \\
\hline
\end{tabular}

*, statistically significant. BMI, body mass index; PSA, prostate specific antigen; NLR, neutrophil to lymphocyte ratio; PLR, platelets to lymphocyte ratio; LMR, lymphocyteto monocyte ratio; SVI, seminal vesicle invasion. 
Table 3 Univariate and multivariate analysis for apical PSMs

\begin{tabular}{|c|c|c|c|c|c|c|}
\hline Variables & \multicolumn{3}{|c|}{ Univariate analysis } & \multicolumn{3}{|c|}{ Multivariate analysis } \\
\hline Age & 1.039 & $1.001-1.078$ & $0.043^{*}$ & 1.036 & $0.998-1.076$ & 0.062 \\
\hline BMI & 1.001 & 0.913-1.099 & 0.977 & & & \\
\hline PSA & 0.73 & $0.433-1.230$ & 0.237 & & & \\
\hline PLR & 1.004 & $0.998-1.009$ & 0.219 & & & \\
\hline LMR & 0.875 & $0.775-0.988$ & $0.031^{*}$ & 0.881 & $0.779-0.996$ & $0.043^{\star}$ \\
\hline Prognostic nutritional index & 1.001 & $0.954-1.050$ & 0.963 & & & \\
\hline Biopsy Gleason sum ( $\geq 8$ vs. $<8$ ) & 0.805 & $0.478-1.355$ & 0.415 & & & \\
\hline Pathologic Gleason sum ( $\geq 8$ vs. $<8)$ & 0.963 & $0.572-1.620$ & 0.886 & & & \\
\hline
\end{tabular}

*, statistically significant. BMI, body mass index; PSA, prostate specific antigen; NLR, neutrophil to lymphocyte ratio; PLR, platelets to lymphocyte ratio; LMR, lymphocyteto monocyte ratio; SVI, seminal vesicle invasion.

Table 4 Univariate and Multivariate analysis for peripheral PSMs

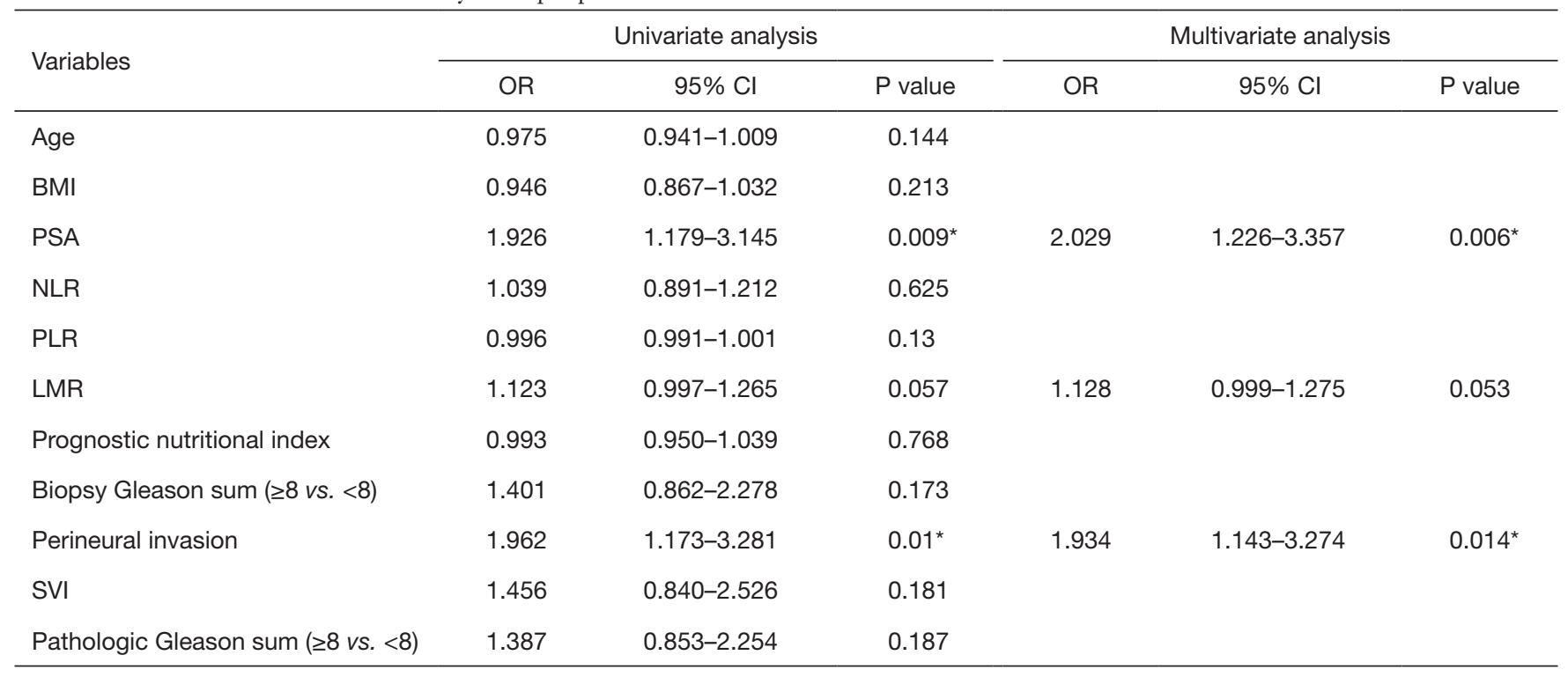

*, statistically significant. BMI, body mass index; PSA, prostate specific antigen; NLR, neutrophil to lymphocyte ratio; PLR, platelets to lymphocyte ratio; LMR, lymphocyteto monocyte ratio; SVI, seminal vesicle invasion.

their association with more robust clinical endpoints is controversial (18). In our research results, we found that preoperative PSA, perineural invasion, and postoperative GS are related to the occurrence of PSMs (all $\mathrm{P}<0.001$ ). Although there is currently no conclusion on the role of preoperative inflammatory factors in the occurrence of PSMs after RP, inflammatory indicators did have a greater impact on the prognosis of PCa. Peng et al. performed a meta-analysis in 2019 and reported that pretreatment LMR, NLR, PLR might be an effective biomarker for poor 
Table 5 Univariate and Multivariate analysis for proximal PSMs

\begin{tabular}{|c|c|c|c|c|c|c|}
\hline Variables & \multicolumn{3}{|c|}{ Univariate analysis } & \multicolumn{3}{|c|}{ Multivariate analysis } \\
\hline Age & 0.99 & $0.955-1.026$ & 0.568 & & & \\
\hline BMI & 0.979 & $0.894-1.072$ & 0.644 & & & \\
\hline PSA & 1.651 & $0.992-2.749$ & 0.054 & 1.436 & $0.847-2.433$ & 0.179 \\
\hline PLR & 0.999 & $0.994-1.003$ & 0.556 & & & \\
\hline LMR & 1.084 & $0.965-1.218$ & 0.173 & & & \\
\hline Prognostic nutritional index & 0.995 & $0.950-1.043$ & 0.846 & & & \\
\hline Biopsy Gleason sum ( $\geq 8$ vs. $<8$ ) & 1.414 & $0.852-2.346$ & 0.181 & & & \\
\hline Pathologic Gleason sum ( $\geq 8$ vs. $<8$ ) & 1.765 & $1.058-2.945$ & $0.03^{*}$ & 1.329 & $0.762-2.317$ & 0.316 \\
\hline
\end{tabular}

*, statistically significant. BMI, body mass index; PSA, prostate specific antigen; NLR, neutrophil to lymphocyte ratio; PLR, platelets to lymphocyte ratio; LMR, lymphocyteto monocyte ratio; SVI, seminal vesicle invasion.

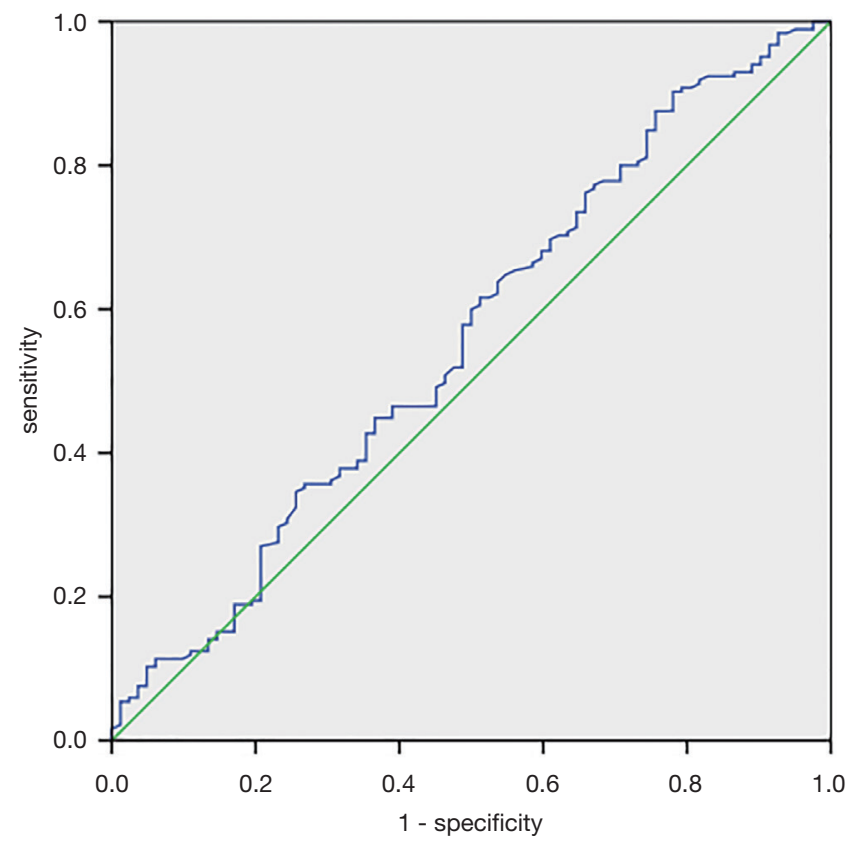

Figure 1 ROC curves in predicting apex PSMs by LMR (continuous, AUC =0.557). ROC receiver operating characteristic, AUC area under the curve.

prognosis in patients with $\mathrm{PCa}$ (19). And Zhou et al. also suggested that LMR played a significant role in initial PCa diagnosis (20). These articles showed that LMR played an important role in the occurrence and development of $\mathrm{PCa}$. In our research, we found that preoperative PSA, LMR, and postoperative perineural invasion, PGS can be used as independent factors to predict the incidence risk of PSMs after RP. And also, in terms of predicting the location of PSMs, LMR may have an inverse relationship with the apical PSMs $(\mathrm{P}=0.043)$. The lower the LMR before surgery, the higher the probability of apical PSMs. Shigeta et al. also showed that high monocyte count predicts poor clinical outcomes in PCa patients. This conclusion indicated that lower LMR may have a worse prognosis in PCa patients (21). At the same time, Lian et al. found that patients with apical PSMs had a higher risk of biochemical recurrence than patients with other PSMs in 2020 (22). These findings lead us to speculate that the impact of LMR on the prognosis of PCa patients may be caused by the incidence of apical PSMs after RP. However, we found that higher LMR may have a greater risk of PSMs. This is contrary to our hypothesis. The reason for this contradiction is still unclear. It may be related to the small number of the population included in this study. Besides, we also found that LMR may be used as an independent predictor of multifocal PSMs. Coelho et al. reported that the clinical stage was the only independent variable that was associated with PSM after RARP (23). They did not find a positive correlation between preoperative PSA and PSMs. Porcaro et al. collected 476 PCa patients after RP, and they also demonstrated that 
Table 6 Univariate and Multivariate analysis for focal PSMs

\begin{tabular}{|c|c|c|c|c|c|c|}
\hline Variables & \multicolumn{3}{|c|}{ Univariate analysis } & \multicolumn{3}{|c|}{ Multivariate analysis } \\
\hline Age & 1.001 & $0.971-1.032$ & 0.961 & & & \\
\hline BMI & 1.052 & $0.979-1.132$ & 0.168 & & & \\
\hline PSA & 2.721 & $1.748-4.235$ & $<0.001^{*}$ & 2.374 & $1.482-3.803$ & $<0.001^{*}$ \\
\hline PLR & 0.998 & $0.995-1.002$ & 0.359 & & & \\
\hline LMR & 1.065 & $0.964-1.178$ & 0.217 & & & \\
\hline Prognostic nutritional index & 1.024 & $0.982-1.067$ & 0.272 & & & \\
\hline Biopsy Gleason sum ( $\geq 8$ vs. $<8$ ) & 1.73 & $1.140-2.626$ & $0.01^{\star}$ & 0.966 & $0.571-1.635$ & 0.897 \\
\hline Pathologic Gleason sum ( $\geq 8$ vs. $<8$ ) & 2.4 & $1.547-3.721$ & $<0.001^{\star}$ & 1.780 & $1.032-3.076$ & 0.039 \\
\hline
\end{tabular}

*, statistically significant. BMI, body mass index; PSA, prostate specific antigen; NLR, neutrophil to lymphocyte ratio; PLR, platelets to lymphocyte ratio; LMR, Iymphocyteto monocyte ratio; SVI, seminal vesicle invasion.

Table 7 Univariate and Multivariate analysis for multifocal PSMs

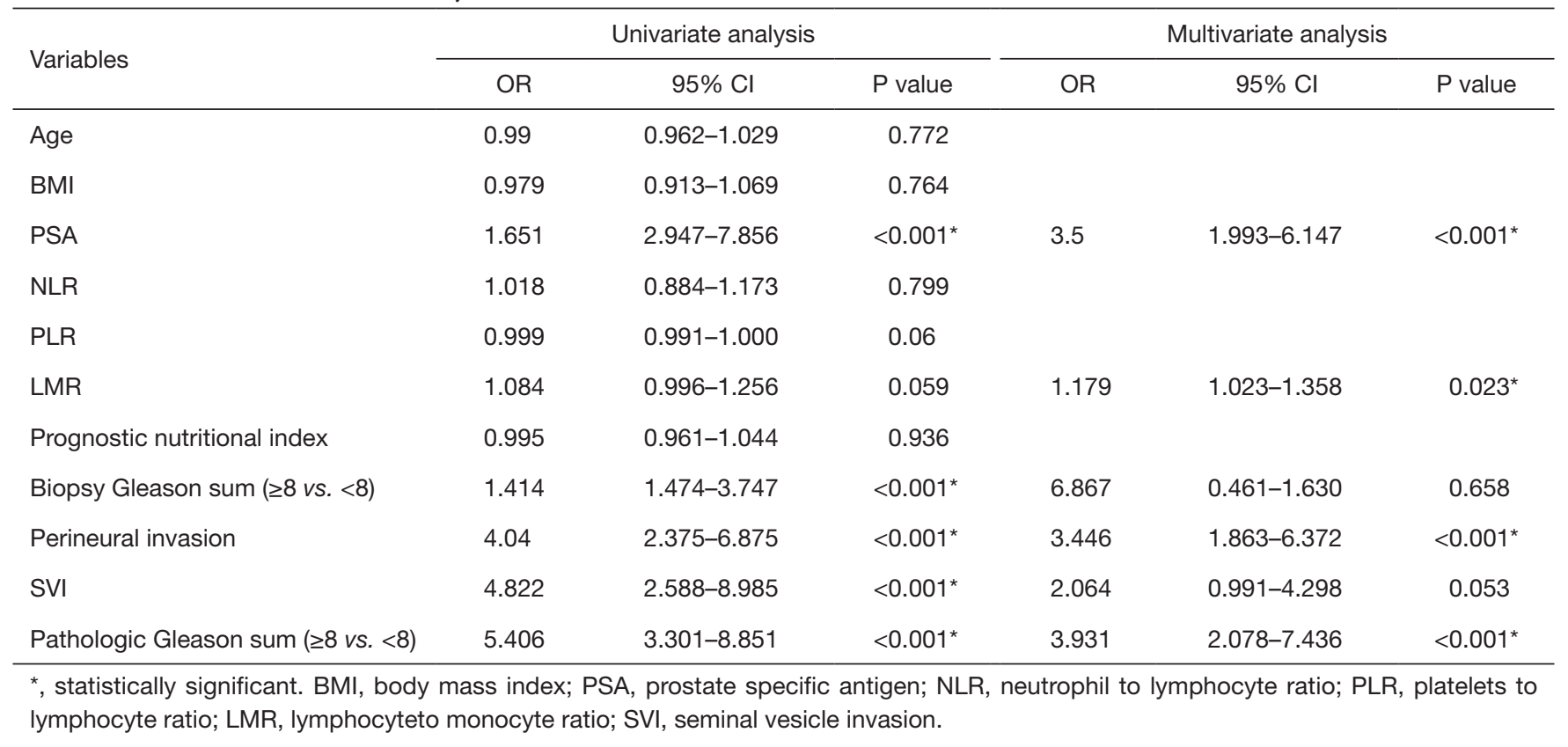

preoperative PSA could not be an independent factor to predict the incidence of PSMs (24). Pooli et al. published an article in 2019 and reported that age and PSA were significantly associated with PSMs rate (25). In summary, the relationship between PSA and PSM is still controversial.
Jayachandran et al. discovered that obesity was associated with an increased risk of overall PSMs. They also suggested that most obese men had a higher risk of apical PSMs (26). Although Coelho et al. did not found that there was a relationship between BMI and the risk of any PSMs, they 
also believe that there was a statistical relationship between BMI and apical PSMs (23). Zilberman et al. thought although BMI was associated with advanced PCa, BMI did not show a correlation in the incidence rate and location of PSMs (27). In our research, we did not find an important association between BMI and incidence rate and the location of PSMs. Currently, the impact of BMI on PSMs after RP remains controversial.

\section{Limitation}

There were a few limitations to our study. First, our research was a retrospective study, so some information or data may be biased. Besides, other inflammatory factors may also affect the results of the study, but we did not include these factors. Furthermore, from our research, the incidence of PSMs in our population was more than $50 \%$, which may also be caused by the experience of the surgeon and technical limitations.

\section{Conclusions}

In general, LMR could be used as a significant factor to predict the incidence of PSMs. Regarding PSMs location, LMR could be used as an independent variable to negatively correlate with the apical PSMs. Besides, LMR was the only preoperative variable independently associated with multifocal PSMs

\section{Acknowledgments}

We would like to thank Tao Li and Shuai Xia for their work in the revision of this article and data extraction.

Funding: Tianjin Science and technology committee (19ZXDBSY00050).

\section{Footnote}

Reporting Checklist: The authors have completed the STROBE reporting checklist. Available at http://dx.doi. org/10.21037/tau-20-1447

Data Sharing Statement: Available at http://dx.doi. org/10.21037/tau-20-1447

Conflicts of Interest: All authors have completed the ICMJE uniform disclosure form (available at http://dx.doi. org/10.21037/tau-20-1447). The authors have no conflicts of interest to declare.

Ethical Statement: The authors are accountable for all aspects of the work in ensuring that questions related to the accuracy or integrity of any part of the work are appropriately investigated and resolved. The study was conducted in accordance with the Declaration of Helsinki (as revised in 2013). The study was approved by the Regional Ethical Review Board in Tianjin medical university second hospital and individual consent for this retrospective analysis was waived.

Open Access Statement: This is an Open Access article distributed in accordance with the Creative Commons Attribution-NonCommercial-NoDerivs 4.0 International License (CC BY-NC-ND 4.0), which permits the noncommercial replication and distribution of the article with the strict proviso that no changes or edits are made and the original work is properly cited (including links to both the formal publication through the relevant DOI and the license). See: https://creativecommons.org/licenses/by-nc-nd/4.0/.

\section{References}

1. Siegel RL, Miller KD, Jemal A. Cancer statistics, 2018. CA Cancer J Clin 2018;68:7-30.

2. Andriole GL, Catalona WJ. The Diagnosis and Treatment of Prostate Cancer: A Review. Ann Rev Med 1991;42:9.

3. Bolla M, van Poppel H, Tombal B, et al. Postoperative radiotherapy after radical prostatectomy for highrisk prostate cancer: long-term results of a randomised controlled trial (EORTC trial 22911). Lancet 2012;380:2018-27.

4. Wiegel T, Bottke D, Steiner U, et al. Phase III postoperative adjuvant radiotherapy after radical prostatectomy compared with radical prostatectomy alone in $\mathrm{pT} 3$ prostate cancer with postoperative undetectable prostate-specific antigen: ARO 96-02/AUO AP 09/95. J Clin Oncol 2009;27:2924-30.

5. Thompson IM Jr, Tangen CM, Paradelo J, et al. Adjuvant radiotherapy for pathologically advanced prostate cancer: a randomized clinical trial. JAMA 2006;296:2329-35.

6. Hong YM, Hu JC, Paciorek AT, et al. Impact of radical prostatectomy positive surgical margins on fear of cancer recurrence: results from CaPSURE. Urol Oncol 2010;28:268-73. 
7. Mantovani A, Allavena P, Sica A, et al. Cancer-related inflammation. Nature 2008;454:436-44.

8. Nishi H, Iga N, Shimizu N. Comparison of the prognostic value of inflammation-based prognostic scores in surgically treated patients with malignant pleural mesothelioma. J STAGE 2016;30:669-79.

9. Okuno M, Ebata T, Yokoyama Y, et al. Evaluation of inflammation-based prognostic scores in patients undergoing hepatobiliary resection for perihilar cholangiocarcinoma. J Gastroenterol 2016;51:153-61.

10. Templeton AJ, McNamara MG, Šeruga B, et al. Prognostic role of neutrophil-to-lymphocyte ratio in solid tumors: a systematic review and meta-analysis. J Natl Cancer Inst 2014;106:dju124.

11. Templeton AJ, Ace O, McNamara MG, et al. Prognostic role of platelet to lymphocyte ratio in solid tumors: a systematic review and meta-analysis. Cancer Epidemiol Biomarkers Prev 2014;23:1204-12.

12. Chan JC, Chan DL, Diakos CI, et al. The Lymphocyte-toMonocyte Ratio is a Superior Predictor of Overall Survival in Comparison to Established Biomarkers of Resectable Colorectal Cancer. Ann Surg 2017;265:539-46.

13. Eastham JA, Kuroiwa K, Ohori M, et al. Prognostic significance of location of positive margins in radical prostatectomy specimens. Urology 2007;70:965-9.

14. Yossepowitch O, Bjartell A, Eastham JA, et al. Positive surgical margins in radical prostatectomy: outlining the problem and its long-term consequences. Eur Urol 2009;55:87-99.

15. Rocco B, de Cobelli O, Rocco F. Re: An evaluation of the decreasing incidence of positive surgical margins in a large retropubic prostatectomy series. J Urol 2004;172:776.

16. Alchin DR, Murphy D, Lawrentschuk N. Predicting the risk of positive surgical margins following robotic-assisted radical prostatectomy. Minerva Urol Nefrol 2017;69:56-62.

17. Patel VR, Coelho RF, Rocco B, et al. Positive surgical margins after robotic assisted radical prostatectomy: a multi-institutional study. J Urol 2011;186:511-6.

18. Yossepowitch O, Briganti A, Eastham JA, et al. Positive surgical margins after radical prostatectomy: a systematic review and contemporary update. Eur Urol 2014;65:303-13.

19. Peng H, Luo X. Prognostic significance of elevated pretreatment systemic inflammatory markers for patients with prostate cancer: a meta-analysis. Cancer Cell Int 2019;19:70.

20. Zhou ZH, Liu F, Wang WJ, et al. Development and validation of a nomogram including lymphocyte-tomonocyte ratio for initial prostate biopsy: a double-center retrospective study. Asian J Androl 2021;23:41-6.

21. Shigeta K, Kosaka T, Kitano S, et al. High Absolute Monocyte Count Predicts Poor Clinical Outcome in Patients with Castration-Resistant Prostate Cancer Treated with Docetaxel Chemotherapy. Ann Surg Oncol 2016;23:4115-22.

22. Lian Z, Zhang H, He Z, et al. Impact of positive surgical margin location and perineural invasion on biochemical recurrence in patients undergoing radical prostatectomy. World J Surg Oncol 2020;18:201.

23. Coelho RF, Chauhan S, Orvieto MA, et al. Predictive factors for positive surgical margins and their locations after robot-assisted laparoscopic radical prostatectomy. Eur Urol 2010;57:1022-9.

24. Porcaro AB, Tafuri A, Sebben M, et al. Positive Association between Preoperative Total Testosterone Levels and Risk of Positive Surgical Margins by Prostate Cancer: Results in 476 Consecutive Patients Treated Only by Radical Prostatectomy. Urol Int 2018;101:38-46.

25. Pooli A, Salmasi A, Johnson DC, et al. Positive surgical margins at radical prostatectomy in the United States: Institutional variations and predictive factors. Urol Oncol 2020;38:1.e17-23.

26. Jayachandran J, Aronson WJ, Terris MK, et al. Obesity and positive surgical margins by anatomic location after radical prostatectomy: results from the Shared Equal Access Regional Cancer Hospital database. BJU Int 2008;102:964-8.

27. Zilberman DE, Tsivian M, Yong D, et al. Does body mass index have an impact on the rate and location of positive surgical margins following robot assisted radical prostatectomy? Urol Oncol 2012;30:790-3.
Cite this article as: Zhou J, Liu R. Positive association between preoperative lymphocyte-to-monocyte ratio and risk of the status of positive surgical margins by prostate cancer: results in 497 consecutive patients treated only by radical prostatectomy. Transl Androl Urol 2021;10(3):1133-1142. doi: 10.21037/tau-201447 